\title{
LE DANUBE VU PAR UN FRANCAAIS
}

Katarina V. Melić, Faculté de philologie et des arts, Université de Kragujevac, katarinamelic@yahoo.fr

10.31902/fll.29.2019.5

UDK 725.182(282.243.7)(497)

UDK 821.133.1-922

Résumé : Le Danube, le plus ancien voyageur européen, est le seul des grands fleuves à couler d'Ouest en Est et à passer à travers dix pays. Qualifié d'« empereur parmi les empereurs " par Napoléon, il a été la source d'inspiration de nombreux artistes: écrivains, peintres, poètes, voyageurs-écrivains... Notre intention est d'étudier comment un artiste-écrivain-voyageur au XIX siècle, Dieudonné Auguste Lancelot, voyageant dans les Balkans le long du Danube, impressionné par ce fleuve et les villes-forteresses qui se trouvent sur ses rives, note et représente à l'aide de mots et d'images ce qui a particulièrement attiré son attention. Nous étudions dans le récit de Lancelot qui voyage par voie fluviale, deux aspects qui portent sur le Danube : les villes-forteresses et le commerce /le tourisme.

Mots-clés: Danube, villes-forteresses, Golubac, commerce, tourisme

L'intérêt des artistes occidentaux pour l'Orient ne date pas du XIXe siècle : déjà dans les Lettres persanes de Montesquieu (1721), se fait sentir l'attrait pour I'Orient. Les événements politiques au XIXe siècle, comme l'expédition d'Égypte de Bonaparte, les débuts de la colonisation en Algérie et les crises d'Orient successives font que l'on s'intéresse à des régions auparavant peu connues. Une nouvelle sensibilité, développée au XIXe siècle, associe le romantisme à l'Orient ; on est à la recherche du dépaysement, de l'étranger et de l'exotique. Le voyage en Orient devient une étape obligatoire de l'initiation artistique et spirituelle.

De plus, l'amélioration des moyens de transport et l'arrivée du bateau à vapeur permettent à de nombreux écrivains et peintres de se rendre eux-mêmes en Orient. Le voyage par voie fluviale est le signe de la nouvelle époque de I'industrialisation en Europe et du progrès technique et scientifique. Le nombre de voyageurs français qui traversent les Balkans augmente considérablement au $X I X^{e}$ siècle. C'est le XIXe siècle qui voit l'avènement d'une nouvelle sorte de voyageurs, celle de l'écrivain qui voyage d'abord pour lui-même. Le voyage devient littéraire tandis que la destination à la mode est le voyage en Orient.

Dieudonné Auguste Lancelot est un de ces artistes-écrivains-voyageurs, oublié par le temps, qui a voyagé dans les Balkans, suivant le cours du Danube et consigné dans ses écrits et dessins, ses impressions sur ces contrées dans la deuxième moitié du XIXe siècle. Dans ce travail, nous nous concentrerons sur ce que Lancelot a noté de son passage en Serbie, suivant le cours du Danube qui est une sorte de fil conducteur, faisant ainsi découvrir les paysages de la Serbie, et plus précisément, les forteresses sur le Danube au lecteur. 


\section{Les Balkans et le Danube}

Pendant des siècles les Balkans ont représenté une énigme. Longtemps restés méconnus, les Balkans sont des territoires montagneux impénétrables, pleins d'obstacles naturels, comme les massifs carpatiques, les forêts sauvages, etc. Les voyageurs visitent les Balkans à partir du XIV siècle, mais une découverte ne commence qu'au XVIII ${ }^{\mathrm{e}}$ siècle. D'après les témoignages écrits, les voyageurs ont trouvé dans les Balkans des peuples qui vivaient différemment. La variété et la dissemblance leur ont paru choquantes. Si au début, on constate surtout une appréciation négative, les opinions changent petit à petit. II faut aussi rappeler les intérêts qui ont emmené les voyageurs aux Balkans ; tout d'abord, c'est la nécessité de connaître de plus près l'ennemi, l'Empire ottoman, et plus tard, admirer sa force et ses victoires en Europe, et finalement, beaucoup plus tard, critiquer le despotisme de cet empire face aux peuples balkaniques.

Comme le plus grand fleuve d'Europe, le Danube (Istros, Mataos, Danubius, Danube, Donau, Duna, Tuna, Dunarea, ...) est le plus ancien voyageur européen. Deuxième fleuve d'Europe par sa longueur, le Danube est le seul des grands fleuves à couler d'Ouest en Est et à traverser dix pays. Né dans la forêt Noire, le Danube va mourir dans la mer Noire. Les voyageurs peuvent se familiariser avec des sites géographiques, culturels, des diversités ethnique et religieuse dans une zone relativement petite partagée entre deux empires, autrichien et ottoman. Un autre aspect de l'expérience danubienne réside dans l'élément oriental et exotique, qui assure également les spécificités culturelles et naturelles du Danube. Longer les rives du Danube offre la possibilité de penser avec la mentalité de nombreux peuples.

Pour Napoléon, le Danube était « l'empereur parmi les empereurs », tandis que l'écrivain médiéval, Constantin le Philosophe, le considère comme l'un des quatre fleuves paradisiaques du monde. À Rome, la fontaine du Bernin est ornée de quatre figures de fleuve, chacun d'entre eux symbole d'une partie du monde. C'est le Danube qui représente l'Europe. En tant que plus grand fleuve navigable en Europe, le Danube a lié et lie encore les peuples, les cultures, les religions, les rites et les contrées, les mythes et les légendes. Le Danube était et reste, pour tous les peuples installés sur ses rives, la source de vie et d'existence, tout comme il a été le témoin de leurs destins historiques, des guerres, des souffrances et des conquêtes. Autrefois, c'était les guerriers, les voyageurs, les douaniers qui naviguaient sur le Danube ; aujourd'hui, ce sont principalement les touristes qui s'embarquent en croisières pour de nouvelles aventures de Vienne jusqu'à la mer Noire. Chemin faisant, ils font connaissance de l'exceptionnelle diversité géographique, culturelle et mythologique des peuples sur le Danube, gastronomique et artistique, des sites archéologiques et des monuments historiques, et surtout, des forteresses et des fortifications. Les forte- 
resses étaient aussi bien construites par les souverains serbes que par les souverains hongrois ou turcs car il n'était possible de développer toute forme de vie et de commerce que dans des endroits fortifiés. Les plus impressionnantes forteresses en Serbie ont été justement construites sur les rives du Danube et sont classées parmi les plus importants monuments européens d'architecture militaire. Des vingt-neuf forteresses et ruines des fortifications en Serbie, sept se trouvent sur les rives du Danube.

Le Danube n'en a pas moins été la source éternelle d'inspiration, de sagesse et d'expérience de nombreux artistes : écrivains, peintres, poètes, voyageurs-écrivains. Il est tout à fait naturel que le Danube devienne un sujet d'écriture et un thème inépuisable pour les nombreux voyageurs français qui visitent ces contrées. Dieudonné Auguste Lancelot est un de ces artistes-écrivains-voyageurs qui a voyagé dans les Balkans suivant le cours du Danube et consigné dans ses écrits et ses dessins, ses impressions.

\section{Dieudonné Auguste Lancelot}

Dieudonné Auguste Lancelot ${ }^{1}$, lithographe, graveur et illustrateur français, est né à Sézanne en 1822 et mort à Paris en 1894. Élève d'Anne-François Arnaud, il expose au Salon épisodiquement, de 1853 à 1876. Il illustre les œuvres des écrivains contemporains et des revues comme Le Tour du monde, Le Magasin pittoresque, Le Monde illustré, Les Jardins, Le Musée français, et autres. Collaborateur du Tour du Monde, il parcourt presque le monde entier - de l'Asie jusqu'en Amérique du Nord en passant par l'Europe et l'Afrique du Nord.

"Le voyage de Paris à Bucharest » est le titre du reportage que Lancelot commence en 1861. Lancelot entreprend en 1860 un voyage avec Victor Duruy, historien et écrivain bien connu, qui les mène de Paris, via Bucarest, à Timisoara. Victor Duruy est chargé d'en faire la relation dans Le Tour du Monde. Mais il devient ministre de l'Instruction publique, en 1863 sous Napoléon III, et Lancelot est invité à écrire le reste de la relation, pour l'itinéraire de Presbourg (Bratislava) à Bucharest. La navigation sur le Danube promet un voyage passionnant et varié de Vienne à la mer Noire. Lancelot note les impressions de son voyage sur le Danube, en bateau de Vienne à Craiova, puis à terre jusqu' à Timisoara. Pour atteindre les Slaves de Turquie, sujet d'étude préféré des voyageurs français, il faut prendre la route par le Danube à partir de Vienne, et passant par Budapest, arriver à Novi Sad, ensuite à Zemun, ville frontalière de la Hongrie, et finalement, à Belgrade qui se trouve de l'autre côté du Danube. Naviguant sur le Danube, Lancelot fait découvrir, par ses mots et ses dessins, les magnifiques contrées de la Serbie de l'époque. L'importance de la description du voyage en bateau tient du fait que l'écriture et le visuel se complètent parfaitement. Ce

\footnotetext{
${ }^{1}$ Pour plus de détails sur Dieudonné Auguste Lancelot et Le Tour du Monde, voir Melić 2016.
} 
témoignage nous apprend comment le public français découvre grâce à la littérature de voyage, des régions moins connues et moins explorées en Europe et se trouvant le long du Danube.

Dans la rédaction de son voyage, Lancelot se positionne en artiste, se décrivant toujours l'album sous le bras et le crayon à la main². II focalise son intérêt sur tout ce qui peut relever de la catégorie du pittoresque : «[...] cette scène d'un air distrait, ne manquait pas d'un charme pittoresque. " (Lancelot, 1865 : 61). Dans son sens plus large, la notion de pittoresque désigne toute description ou représentation évocatrice des caractéristiques d'un pays et son " aspect attrayant, curieux, typique » :

Rappelons que pittoresque signifie étymologiquement : " qui est digne d'être peint ", qui est susceptible de constituer un motif (objet, situation, cadre, personnage) dont un peintre pourrait tirer parti dans une scène de genre, dans laquelle il aurait fonction d'indice (par exemple : le motif du voile suffit à indiquer la scène orientale). Par extension, le mot " pittoresque " désigne en peinture et en littérature au XIXe siècle tout traitement d'une scène qui procède par l'insistance sur quelques motifs typiques (faisant référence à un type) qui apportent au lecteur le dépaysement qu'il recherche (car le pittoresque implique l'éloignement, l'extériorité) (Barthèlemy, 2018: 11).

Pour Lancelot, le pittoresque relève aussi bien du monde que du regard du voyageur et de son parti pris de le découvrir ou de l'attribuer à certaines choses. Le pittoresque peut être évident et réel : il peut résulter de la façon de voir et de l'appréciation du voyageur - si on le recherche, on finit par le trouver, ou, à le créer.

\section{Le Danube et la nature}

Lors de son voyage, suivant le cours du Danube, Lancelot passe par Apathin, Neusatz, Petrovaradin, Semlin ou Zemlin, Pancsova, Belgrade, Grodska, Semendria, Rama, Golumbacz, Cladovo, les Portes de Fer. Plongé dans la description et le désir de rapprocher ces contrées inconnues et mystérieuses au lecteur français, il fait des relations inspirées et pittoresques de tous ses passages et de ses escales. Les descriptions sont nombreuses et détaillées. Comme le pittoresque est envisagé et recherché, très souvent, les réalités sur place sont réduites au curieux et à l'étrange. Comme Lancelot est parti en voyage avec l'intention de voir le pittoresque, il le trouve un peu partout. Lancelot fait des croquis des réalités locales rencontrées qu'il veut faire admirer par le lecteur

\footnotetext{
${ }^{2}$ "C'était, certes, un croquis à faire » (Lancelot, $1865: 61$ ).
} 
français. Lui-même les admire, étonné devant ce qui est surprenant et imprévu, et son admiration valorise ces réalités dans la catégorie du pittoresque :

Cette rivière, et une sentinelle qui, nonchalamment appuyée à un tronc d'arbre, contemplait cette scène d'un air distrait, ne manquait pas d'un certain charme pittoresque. C'était, certes, un croquis à faire (Lancelot, 1865 : 61).

Ses dessins de paysage, et surtout leur nombre, montrent qu'il est toujours à la recherche du pittoresque. Le texte le confirme, en décrivant longuement tous les lieux : les montagnes escarpées et les ravins étroits, les eaux du Danube et les lieux isolés et désolés, les masses granitiques et les rochers escarpés :

De Peterwardein à Belgrade, la rive droite présente une succession de hautes croupes argileuses séparées par des ravins qui descendent du sommet, si étroits, qu'on les croirait creusés de mains d'hommes. Quelquesuns de ces ravins forment chemin ; mais quel chemin! Des ornières à y coucher une locomotive (Lancelot, 1865 : 58).

On peut le lire dans la description des ruines de la forteresse de Rama :

À un coude brusque du Danube, s'avance, comme pour lui barrer le passage, un écueil portant les ruines, encore imposantes, d'un ancien château fort que couronne un donjon très-élevé. "C'est Rama ", me dit un vieux marin, qui depuis 1835 navigue sur le Danube et qui connaît à fond l'histoire de sa navigation encouragée à regret d'abord par l'Autriche et vue d'un mauvais œil par la Turquie (Lancelot, 1865 : 79).



<http://gallica.bnf.fr/ark:/12148/bpt6k34386x.r=> 
La forteresse de Smederevo (Semendria) est aussi décrite dans sa majestuosité :

La forteresse de Semendria, bâtie en 1433, par George Brankovitch, le dernier despote serbe, est superbe comme décoration. S'avançant comme un promontoire jusqu'au milieu du fleuve, très large en cet endroit, elle présente de face au courant un rempart et une haute muraille crénelée, sur laquelle se dressent vingt-sept tours carrées. [...] Les tours sont fendues, les créneaux édentés, et tout cela n'a que juste le degré de solidité nécessaire à une décoration (Lancelot, 1865 : 78).

Il est toujours à la recherche du pittoresque dans la description du chemin de Trajan et du pont de Trajan :

Ceux qui ont creusé ce chemin sans points d'appui, n'avaient pas la vapeur pour les transporter ou pour forer la pierre, ni la poudre pour fendre les lourdes assises du roc, et les précipiter dans le fleuve. Ils ne savaient pas se faire obéir de la force aveugle, la contenir et la diriger ; mais ils étaient eux-mêmes une force intelligente. Réfléchissez un peu à ce qu'ont fait et nous ont laissé les anciens, comparez la faiblesse de leurs moyens et la grandeur de leurs œuvres (Lancelot, 1865 : 81).

Vues du milieu du fleuve, elles présentent sur chaque rive exactement la même figure. On dirait deux grands sièges de pierre posés au bord de l'eau et se faisant face. Un grand pan de maçonnerie surmontant un massif carré, que le temps a arrondi, figure régulièrement le dossier. II me semble que les deux colosses de la plaine de Thèbes y seraient assis à l'aise. Construit par l'architecte Apollidore de Damas, sur l'ordre de Trajan, lorsqu'il entreprit sa seconde campagne contre Decebale, il se composait de vingt arches de cent cinquante pieds de hauteur, présentant d'une pile à l'autre une ouverture de soixante pieds. Sa largeur était également de soixante pieds, et sa longueur de neuf cents. Cette œuvre hardie n'eut pas une longue durée. Trajan avait fait construire ce pont pour passer en Dacie ; son successeur jugea prudent de le renverser, parce que les Barbares, à leur tour, pouvaient s'en servir pour envahir le territoire romain (Lancelot, 1865 : 92).

Ce sont des vestiges de l'histoire et de la nature qu'il découvre en suivant le Danube. Le Danube est son fil conducteur, il préfère ce moyen de voyage car il lui permet de voyager au fil de ses désirs, intuitions, etc. Le dessinateur est fasciné par le spectacle de la nature qui présente partout des sites admirables: 
Les accidents de la nature, quels qu'ils soient, n'éveillent d'ordinaire en moi qu'un sentiment irréfléchi d'admiration accompagné d'un désir irrésistible d'aller en avant, pour reporter ma vue sur de nouveaux objets (Lancelot, 1865 : 88).

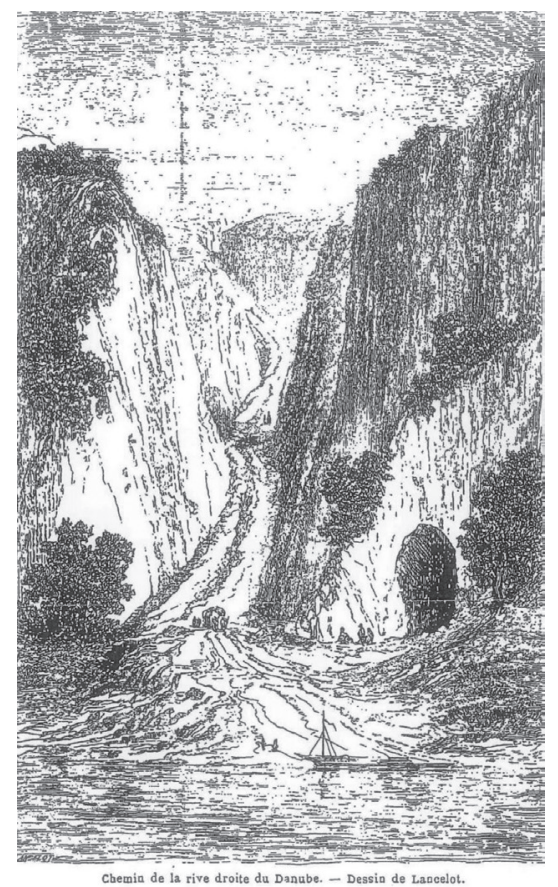

<http://gallica.bnf.fr/ark:/12148/bpt6k34386x.r=>

Dans la description de ces paysages, le pittoresque révèle tout son sens : le mot désigne des réalités qui sont dignes d'être peintes ou qui, par leurs formes et leurs couleurs, par leurs contrastes. Nous ne donnons ici que deux exemples, parmi les nombreux relevés par Lancelot :

Aux moulins de Bezdan, succèdent les pêcheries d'Apathin. Les pêcheries forment un véritable village bâti en pleine eau, et qui par la singularité de son aspect, fait une heureuse diversion à la monotonie du paysage.

Qu'on se figure un fouillis de constructions en bois, cabanes, huttes, hangars, guérites, appentis de branchages et de paillis posés sur des charpentes. Au milieu une place marquée par un grand mât. A chaque pieu, à chaque pilotis, à chaque saillie tient la corde d'un filet tendu. Sous les toits, à toutes les portes, une nacelle est amarrée, et à travers chaque interstice par où l'œil peut glisser, le long des ruelles, par-dessus les légères toitures, l'on voit courir des barques dont les conducteurs, hommes ou femmes, pagayent debout (Lancelot, 1865 : 57). 
Voici ce qu’il écrit sur Semlin :

Deux ou trois ruelles conduisent du débarcadère de Semlin à la ville proprement dite, qu'on aperçoit, en face et à droite, à une distance respectueuse du fleuve, car le Danube est un voisin mal commode, et il ne fait pas bon toujours le serrer de trop près. À gauche, s'étend une prairie marécageuse plantée d'arbres et bordée de maisons de pauvre apparence. Semlin, situé au confluent du Danube et de la Save, n'est séparé de Belgrade que par la largeur de la rivière, très-spacieuse, il est vrai, en cet endroit (Lancelot, 1865 : 59).

Dans le cas des paysages, dont certains lui plaisent parce qu'ils correspondent à ce qu'il avait déjà vu, d'autres à ses attentes soit romantiques soit d'orientalité. D'ailleurs, l'utilisation fréquente d'adjectifs dramatiques: grandiose, isolé, défilé romantique, vilains méchants, forêts anciennes, etc. résume l'idée romantique de la nature ici écrite dans le paysage du Danube. Malgré la nécessité de montrer la vraie nature de la nature, les artistes et les écrivains de voyage du XIXe siècle recherchent continuellement des sites inhabituels et exotiques, tandis que les phénomènes naturels sont pour eux l'essence de la personnification des drames, des mystères, des mythes, des dangers et de la majestuosité des lieux, et de l'histoire. II est notoire que le sublime a largement façonné l'image de la nature du XIXe siècle, même lorsque ce tableau aurait dû être une vue objective. Pour cette raison, nous nous trouvons dans la nature de la splendeur exaltée sous deux formes: dans les phénomènes naturels et dans les représentations de ruines qui rappellent la supériorité de la nature sur l'homme.

Plusieurs toponymes le long du Danube avaient une signification particulière - ce sont, par exemple, les ruines de la forteresse de Golumbacz ${ }^{3}$, le rocher de Babakaj. À l'entrée du défilé de Djerdap, dans les méandres du Danube et sur les hautes falaises, la forteresse de Golubac, symbole des guerres médiévales

\footnotetext{
${ }^{3}$ Selon la légende, la construction de la forteresse de Golubac est liée à Prokleta Jerina/Irène la damnée, princesse byzantine (Irène Kantakouzène) et épouse du despote Đurađ Branković. Haïe par le peuple à cause des lourds impôts qu'elle a imposés lors de la construction de la forteresse, elle cultivait des pigeons dans la ville, d'où le nom de Golubac.

4 Du temps de la domination turque, le Pacha acheta une jeune fille du nom de Golubana (golub en serbe - " pigeon ") qu'il fit emmener, avec ses autres esclaves, dans la ville fortifiée sur le Danube. Comme elle était devenue une beauté, le Pacha voulut la faire mettre dans son harem. Pendant la nuit précédant la célébration de cet acte, une grosse tempête se déclencha : la pluie et la grêle tombèrent, le Danube devint trouble et le vent mugit. À cause du mauvais temps, la garde se réfugia dans la tour, et la malheureuse jeune fille, voulant éviter son destin, essaya de descendre dans le fleuve à l'aide de ceintures de soie. Des pêcheurs de l'autre côté du fleuve la virent et la sauvèrent d'une mort certaine. Le lendemain, le Pacha alarma toute la ville pour retrouver son épouse morte ou vive. L'ayant retrouvée, il la fit enchaîner sur un rocher au milieu du Danube. De son balcon, il lui cria de se repentir : « Baba-kaj! » La jeune fille refusa de le faire et resta enchaînée. La ville devrait son nom à cette jeune fille, tout comme le rocher Baba Kaj.
} 
et des revirements sur la frontière danubienne, veille. Au cours de son histoire mouvementée, elle a enfanté des héros, des rois, des sultans, et leurs grandes batailles toutes avec le même but - la conquête de cette forteresse - ville :

À Golumbacz, il me montre une admirable ruine, la plus belle des bords du Danube. Qu'on se figure une pyramide de rochers nus sortant du lit du fleuve et sur laquelle s'entassent de la base au sommet une succession de tours et de donjons reliés entre eux par des chemins couverts et des remparts crénelés, jusqu'à l'extrême pointe couronnée par une tour ronde gigantesque. Du pied de cette tour un des côtés de la pyramide descend jusque dans le fleuve par des degrés de rochers à pic. Une barque mâtée, d'une assez grande dimension, abritée par une échancrure du roc, disparaît dans l'ensemble imposant de ces constructions et de la masse de granit qui les porte. Murailles et rochers, d'une belle teinte rougeâtre uniforme à ce point qu'on les croirait le même bloc, se détachent d'une encoignure de la montagne boisée et coupée par d'énormes crevasses (Lancelot, 1865 : 79).

Lancelot note quelques légendes en prenant bien soin de les relier à l'ambiance naturelle : " Les accidents et les phénomènes naturels de la rive gauche du fleuve ont donné lieu également à une foule de récits merveilleux » (Lancelot, 1865 : 79). Les ruines de la ville médiévale de Golubac et le paysage environnant présentent une concentration de tous les éléments sublimes et pittoresques dans la nature. Golubac possède tous les éléments clés - isolement, grandiosité, effet dramatique - et provoque un sentiment de vénération face à l'écoulement du temps. Comme le montre l'illustration de Lancelot à la page 77 du récit de Lancelot, deux aspects importants ont été fusionnés dans la représentation de la forteresse et de la nature de Golubac : la vaste nature vaste et les ruines dramatiques qui se trouvent en son centre. Cela donne un ton spécial au dessin de Lancelot. L'idée de la magnificence des ruines est particulièrement significative car les ruines permettent un contact avec le passé, c'est-à-dire les monuments du temps. 


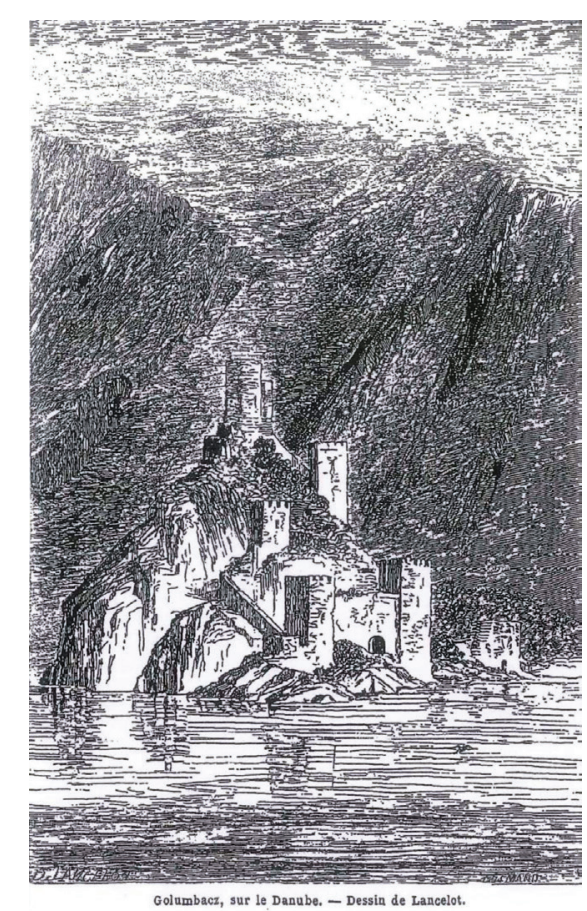

<http://gallica.bnf.fr/ark:/12148/bpt6k34386x.r=>

Un autre concept est important lors de la représentation de ces forteresses - l'idée de la magnificence des ruines. À partir du XVIII siècle, et surtout au XIX ${ }^{e}$ siècle, l'obsession de l'idée des ruines est présente dans la culture et art. Les ruines permettent un contact spécial avec le passé, et en même temps, leurs monumentalisations, et dans le sens le plus restreint - la transformation des artefacts du passé en monuments du temps. La ruine a été un idéal esthétique absolu pour l'homme de l'époque (surtout si elle ressemblait à l'idéal de l'Antiquité), et la glorification des ruines classiques est devenue un idéal esthétique. La ruine rend visible l'attrait historique de la région car elle a incorporé en elle l'idée de l'histoire qui est un des fils conducteurs de Lancelot dans son récit de voyage.

Ceci était d'ailleurs un des idéaux esthétiques romantiques que l'on retrouve tout au long du siècle. Cela est aussi le cas dans les descriptions de Lancelot des autres forteresses sur le Danube : Belgrade, Novi Sad, Smederevo, Ram, Kladovo. 


\section{Le Danube : commerce et tourisme}

Lancelot n'est pas seulement intéressé par la beauté des sites qu'il visite. La construction des ports danubiens, la navigation sur le Danube, qui liait l'Europe centrale et l'Empire ottoman, la possibilité de la construction d'un canal Danube - mer Noire, ont déterminé les gouvernements de la France et de l'Angleterre à être directement intéressés par ces endroits. Les intérêts de la France, de l'Angleterre et de la Russie ne sont pas toujours les mêmes en ce qui concerne les Balkans. Ce qui importe pour l'Angleterre, c'est d'éviter que la Russie ne s'impose dans les Balkans et que la France n'empiète pas sur son empire méditerranéen, à savoir la route des Indes. Elle s'entend avec la France en 1856 pour garantir l'intégrité territoriale de l'Empire ottoman, en échange de quoi le sultan permet l'ouverture de la Mer Noire et du Bas Danube à la navigation et au commerce. Le transport fluvial profite de ce revirement de situation. Pour tout Européen qui désire gagner les rives du Bosphore, il existe, à partir de 1856, une autre possibilité, à savoir le Danube, et c'est surtout la réglementation de la navigation internationale sur le fleuve qui donne un élan au trafic par cette voie. II y a au XIXe siècle un développement considérable des moyens de transports ; on commence à ce moment à se déplacer par bateau. L'apparition des bateaux à vapeurs a favorisé des voyages par voie fluviale et maritime, et ce genre de voyage a eu un effet important sur l'inspiration des voyageurs et des écrivainsvoyageurs, car la navigation est perçue comme un moment de réflexion pour les voyageurs. Les considérations économiques et géostratégiques ont suscité l'intérêt du gouvernement français pour la Serbie. La France regarde favorablement l'acquisition de l'autonomie serbe au sein de l'Empire ottoman et ouvre en 1839 son premier consulat dans une Serbie autonome. Les consuls français dans la principauté de Serbie ont bien observé l'importance de ce petit pays pour le commerce régional. En 1842, le consul de France considère la position géographique de la Serbie comme une position centrale qui permet de relier la mer Noire et la Méditerranée au monde germanique. C'est au début des années 1840 que les premiers liens commerciaux, modestes il est vrai, entre la France et la France s'établissent. Lancelot est certainement bien informé des liens commerciaux entre la France et la Serbie car il n'oublie pas de noter l'importance du Danube dans le commerce :

Le commerce en gros est considérable. Belgrade est le grand entrepôt des marchandises à destination non seulement de la Serbie, mais de toutes les provinces turques limitrophes, Bulgarie, Albanie, Bosnie. Il fournit à lui seul plus de la moitié de la valeur des importations et des exportations de toute la principauté. Les importations se font par la voie du Danube et de la Save, qu'un service régulier de navigation à vapeur fait communi- 
quer, à Basiach avec les chemins de fer du sud de l'Autriche, à Sissek avec le chemin de fer de l'Adriatique (Lancelot, 1865 : 68).

L'achèvement prochain de la ligne de Sissek à Semlin, l'exécution depuis longtemps projetée de la grande ligne qui, traversant la Serbie dans toute sa longueur, doit relier Vienne à Constantinople, en facilitant les communications et les moyens de transport, auront pour effet d'accroître encore la prospérité commerciale de Belgrade, qui, transformé en port franc, deviendrait bientôt le Hambourg de l’Orient (Lancelot, 1865 : 68).

Les bateaux accélérés qui partent deux fois par semaine de Pesth pour Basiach en correspondance avec la ligne de Londres et de Paris à Constantinople par Kustendjé, mettent vingt-quatre heures pour parcourir cette distance [...] (Lancelot, 1865 : 78).

C'est par la description que les informations et le savoir circule ; elle n'a pas seulement une fonction ornementale, mais joue un rôle de premier plan. Le récit de voyage est aussi un texte didactique qui véhicule des observations et des informations. Une invitation au voyage est certes lancée : c'est ce que Philippe Hamon appelle la « description-recette » (Hamon, 1981 : 102).

Et dernier point de notre réflexion, puisque les récits de Lancelot n'ont pas seulement une mission culturelle et éducatrice, mais aussi une visée touristique, Lancelot écrit en fonction des futurs touristes. Le mot « tourisme » vient du mot anglais tourism qui signifie le voyage circulaire ou le fait de voyager pour le plaisir :

Partir voyager, c'est rompre avec la monotonie de la vie quotidienne. C'est élargir son horizon, explorer le monde, découvrir une autre nature, des peuples différents. C'est respirer le parfum de l'aventure. C'est changer de vie. Voyage et exotisme, deux mots magiques, chargés de promesses et de rêves (Couprie, 1986 : 6).

$\mathrm{Si}$, au XVIII et au début du XIXe siècle, la popularité des récits de voyage était due, en partie à l'impossibilité pour une grande majorité de la population d'effectuer des voyages réels car ils exigeaient de grandes sommes d'argent et des appuis diplomatiques, vers le milieu du XIX siècle, la situation change : I'apparition des bateaux à vapeurs, du chemin de fer et des hôtels, le progrès technique et scientifique qui se propage, la cessation des hostilités contribuent à la facilitation et à la multiplication des voyages. À cette époque naît une sorte de tourisme caractérisée par la sécurité et la prévisibilité du voyage. Ainsi, Lancelot fait l'éloge de la compagnie de transport Lloyd : "J'espérais aussi trouver près 
d'une compagnie française de navigation, tout nouvellement créée et qui avait son siège à Belgrade, la possibilité de continuer mon voyage jusqu'à Giurgevo avec moins de rapidité que sur les bateaux du Lloyd » (Lancelot, 1865 : 65). Le Lloyd autrichien était réputé pour sa bonne organisation de voyages, sa courtoisie, son excellent service et confort, surtout pour les voyages de courte ou moyenne durée. Les voyageurs se déplacent plus rapidement et en plus grande nombre, et avec plus de confort. Les voyageurs ont maintenant à leur disposition des hôtels en nombre croissant, et c'est à l'intention des futurs touristes que Lancelot note ses impressions sur les hôtels en Serbie : « [...] je noterai seulement ce qui suit comme un renseignement utile à ceux de nos lecteurs qui feraient, par aventure, le même voyage " (Lancelot, 1865 : 90). Lancelot nuance ses réflexions et ses opinions : il lui arrive de trouver intéressant ce qu'il aurait peut-être voulu rejeter. On voit bien dans le texte qu'il y a des choses qu'il trouve difficile à accepter, et donc difficile à faire accepter aux lecteurs. Lancelot désamorce les critiques en les faisant dire par d'autres voyageurs : un Italien, un Anglais... À ce but, il fait de nombreuses comparaisons avec d'autres peuples et contrées visités afin de les faire entrer dans un cadre commun : par exemple, la pierre de Trajan ressemble à " une pirogue indienne ". Lancelot veut montrer qu'il n'y a pas en fait que des variations civilisationnelles, tout aussi intéressantes bien que différentes (Melić 2016). Il écrit dans la lignée de la politique du Tour du Monde qui consistait non seulement à susciter la curiosité de son public populaire ou à former son goût esthétique, mais à promouvoir une politique culturelle, ayant pour fonction de populariser différentes cultures.

En conclusion, Lancelot, dans la relation de son voyage sur le Danube pour la revue Le Tour du monde, est fasciné par le Danube ; il décrit sa beauté et sa forme à toute occasion. II s'attarde surtout sur la description de toutes les villesforteresses qu'il rencontre en cours de route. De toutes les forteresses qu'il a décrites et dessinées, nous nous sommes attardés surtout sur la forteresse de Golubac car celle-ci est, non seulement le plus longuement décrite et dessinée par lui dans toute sa majestuosité. Dans ce cas, le visuel complémente les mots. Ses descriptions correspondent à l'attente de l'horizon du lecteur de l'époque, marqué par le mouvement romantique. Le regard de l'artiste se calque sur celui du voyageur et Lancelot revendique cette vision artistique ; au sens figuré, il étend l'emploi du mot pittoresque qu'il reprend constamment en dehors du domaine artistique. Comme la revue pour laquelle il fait sa relation, Le Tour $d u$ monde, se donne pour but de susciter la curiosité du lectorat de l'époque, de populariser des cultures différentes et de sensibiliser l'opinion public à leur égard, Lancelot suit à la lettre les principes de la revue. Le Danube étant son fil conducteur, il ne se borne pas à sa seule description, mais note aussi les possibilités de commerce et de tourisme qu'il offre. La relation du voyage de Lancelot 
n'est pas seulement une invitation au voyage pour le lecteur, mais aussi pour de futurs marchands et commerçants.

\section{Références bibliographiques}

Aurenche, Marie-Laure. Édouard Charton et I'invention du « Magasin Pittoresque » (1833-1870), Paris : Honoré Champion, 2002.

Barthèlemy, Guy. L'image de l'Orient dans le XIX siècle, Paris : Bertrand Lacoste, 1992.

Charton, Édouard. "Préface ", Le Tour du monde, nouveau journal des voyages, 1, 1860, < http://gallica.bnf.fr/ark:/12148/bpt6k34377z>, [consulté le 12 mai 2018]

Bataković, Dušan. Les sources françaises de la démocratie serbe. 1804-1914, Paris : CNRS, 2013.

Couprie, Alain. Voyage et exotisme au XIXe siècle, thèmes et questions d'ensemble, Paris : Hatier, 1986.

Doiron, Normand. "L'art de voyager. Pour une définition du récit de voyage à l'époque classique », Poétique 73, Paris, 1988.

Hamon, Philippe. Du descriptif, Paris : Hachette, 1981.

Lancelot, Auguste Dieudonné. « De Paris à Bucharest, Le Tour du monde, nouveau journal des voyages ", vol. 11/01-06 (1865). <http://gallica.bnf.fr/ ark:/12148/bpt6k34386x.r=>, [consulté le 23 mai 2018]

Magri-Mourgues, Véronique. "L'écrivain-voyageur au XIXe siècle : du récit au parcours initiatique ", Tourisme, voyages et littérature, Cahiers Festival TransMéditerranée. Ed P. Euzière, 2007, 43-54. <https://halshs.archivesouvertes.fr/hal-00596462/document>, [consulté le 19 mai 2018]

Melić, Katarina. " La Serbie au XIXe siècle vue par un artiste-écrivain-voyageur, Auguste Dieudonné Lancelot », Лицеум XXII. 16, Kragujevac, 2016.

Pageaux, Daniel. Littérature générale et comparée, Paris, Armand Colin, 1994.

SRETENOVIĆ, Stanislav. Francuska i Kraljevina Srba, Hrvata i Slovenaca, Beograd, ISI, 2008.

Weber, Anne-Gaëlle. "Le genre romanesque du récit de voyage scientifique au XIX siècle. ", Sociétés \& Représentations, 21, Paris, Sorbonne, 2006. $<$ www.cairn.info/revue-societes-et-representations-2006-1-page-59. htm. >, [consulté le 12 mai 2018] 


\section{THE DANUBE IN THE EYE OF A FRENCHMAN}

The Danube, the oldest European traveller, is the only major river to flow from West to East and pass through ten countries. This river has been the source of inspiration for many artists: writers, painters, poets, travellers-writers. Our intention is to study how an artist-writer-traveller in the nineteenth century, Dieudonné Auguste Lancelot, travelling in the Balkans along the Danube, and impressed by this river and the fortress towns on its shores, notes and represents with words and images what has particularly attracted his attention. He presents a whole range of historical, geographical, ethnographic facts with the aim of popularizing other cultures. In Lancelot's narrative, following the current of the Danube, we study particularly two aspects of the Danube: the cities-fortresses on its shores, and the possibilities of trade and tourism in relation with the opening of the Danube for free navigation and commerce.

Keywords: Danube, the cities-forteresses, Golubac, trade, tourism 